\title{
Phase Sensitive Parametric Mixers for Coherent All-Optical Signal Processing
}

\author{
Joseph Kakande*, Radan Slavík, Periklis Petropoulos and David J. Richardson \\ Optoelectronics Research Centre, University of Southampton, Southampton, SO17 1BJ, UK \\ *ikk@orc.soton.ac.uk
}

\begin{abstract}
We discuss the application of phase sensitive fiber optical parametric devices for the processing and quantization of optical phase including recent experimental demonstrations.
\end{abstract}

\section{Introduction}

The demand for exponential growth in the capacity of fiber optic communication links will have to be met by utilising both advances in optical technologies, such as high coherence sources and low noise amplifiers, as well as breakthroughs in electronic analog-to-digital conversion (ADC) and digital signal processing (DSP). Optical signal processing techniques must be applied judiciously however, with a focus on providing simple, but scalable functions, and by remaining in the optical domain becomes possible to obtain not just high speed, but low latency and power per processed bit. Phase sensitive (PS) optical parametric processes allow direct measurement and manipulation of the optical phase, which is crucial given the increasing relevance of phase modulated data signals.

In a fiber based PS mixer, one or two pump beams coherently interact via four wave mixing (FWM) with a signal (or signal-idler pair). Depending on the relative phase between the interacting waves, the signal experiences either gain or de-amplification, allowing independent control of the I and Q phase quadratures [1]. This process has been used for the regeneration of binary phase encoded (BPSK) signals [1,2], and recently a field trial of a black-box BPSK regenerator highlighted the practicality of such schemes [3]. The functionality of the PS mixer has been radically enhanced by a novel general technique that allows the multi-level all-optical quantization of phase encoded optical signals in a simple, compact and reconfigurable system employing a single nonlinear optical element [4].

\section{Concept and discussion}

In its most general form, a quantizer takes a ramped input and produces a staircase output by adding a sawtooth to an analog ramp. We can synthesize an $M$-level phase staircase by coherently adding to the input signal a phase conjugated harmonic (generated by a cascaded FWM process) bearing a temporal phase modulation $M-1$ times that on the signal, analogous to Fourier synthesis of complex signals [4]. The resulting waveform has its phase quantized to $M$-levels and an amplitude response of periodicity $\pi / M$.

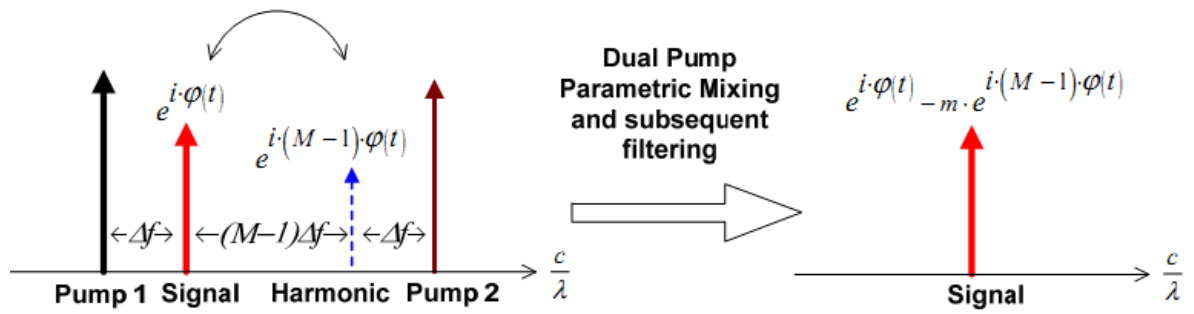

Figure 1: Combination of two pumps with an analytical signal and a conjugated higher order phase harmonic in a PS mixer to synthesize an M-level staircase phase transfer function.

We exploit the efficiency of FWM in HNLF to obtain such a PS mixer by providing both pumps 1 and 2 as well as the signal at the mixer input [5]. The coherent mixing process is then distributed along the fiber length (Fig. 1); initially the cascaded mixing process 
dominates, and then as the harmonic starts to grow in power the coherent conjugated addition takes place. A slow feedback mechanism is required to keep all the waves phase locked.
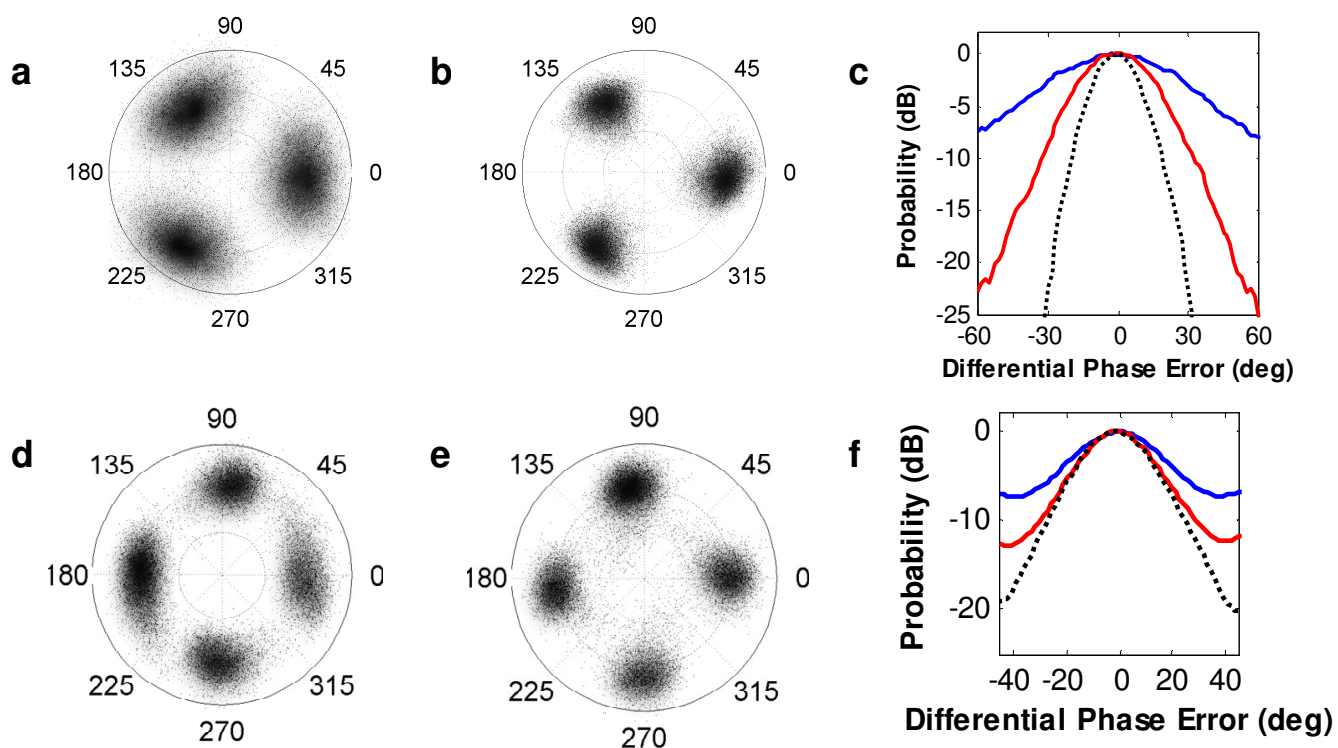

Figure 2: Constellation data when PS mixer is operated as a multilevel phase regenerator with a noisy input signal. a, b, 3-PSK input and output constellations respectively. c, 3-PSK differential (symbol-tosymbol) phase error distribution. d, e, QPSK input and output constellations respectively. f, QPSK differential (symbol-to-symbol) phase error distribution. In the distribution plots (c\&f), blue lines are at regenerator input, red lines at regenerator output and black dotted line is signal without any added noise.

As an example, a PS mixer was operated as a phase quantizer and tested with two modulation formats - 6 Gbaud 3-PSK and 10 Gbaud QPSK While 3-PSK is not currently deployed in communications links due to difficulties in encoding binary electrical data to three levels, this was intended to demonstrate the capability of the scheme to process not just even but also odd levels of modulation. The signals were degraded using both added Gaussian optical noise and nonlinear phase noise emulated with a phase modulator. Following the PS mixer, the phase and amplitude excursions are significantly reduced for 3PSK, Fig. 2b. The differential phase error distribution (symbol-to-symbol phase difference) is shown Fig. 2c. As can be seen, the probability of differential decoded digital errors (when the analog error is over $60^{\circ}$ for 3 -PSK) is reduced by two orders of magnitude. For QPSK, the signal constellations before and after the regenerator are shown in Fig. $2 \mathrm{~d}$ and $2 \mathrm{e}$ respectively. Once again, the differential phase error distributions are plotted, Fig $2 \mathrm{f}$, showing an order of magnitude improvement in symbols that would have been detected as error if a differential receiver had been used (symbols with differential error $>45^{\circ}$ ).

In conclusion, PS mixers now allow for reconfigurable phase quantization, allowing a new class of optical signal processing functions to be exploited. Advances in fiber design and phase locking of high power optical pumps will enhance these capabilities further.

\section{Acknowledgements}

This research has received funding from the European Communities Seventh Framework Programme FP/2007-2013 under grant agreements 224547 (PHASORS).

\section{References}

[1] K. Croussore, et al., "Demonstration of phase-regeneration of DPSK signals based on phase-sensitive amplification," Opt. Express, 13, 3945-3950 (2005).

[2] R. Slavík et al, "All-optical phase and amplitude regenerator for next-generation telecommunications systems," Nature Photonics 4 , 690-695 (2010).

[3] R. Slavík et al, "Field-trial of an all-optical PSK regenerator in a $40 \mathrm{Gbit} / \mathrm{s}, 38$ channel DWDM transmission experiment, "Paper PDPA7, OFC 2011, Los Angeles 6-10, Mar 2011.

[4] J. Kakande et al, "First demonstration of all-optical QPSK signal regeneration in a novel multi-format phase sensitive amplifier," ECOC 2010 Turin 19-23, Italy, PD 3.3, Sep 2010.

[5] J.Kakande et al, "Phase and amplitude regeneration at 56 Gbaud in a novel input-idler-free non-degenerate phase sensitive amplifier", Paper OMT4, OFC 2011, Los Angeles 6-10, Mar 2011. 